\title{
New monatomic layer clusters for advanced catalysis materials
}

\author{
Bin-Wei Zhang ${ }^{1}$, Long Ren ${ }^{1}$, Yun-Xiao Wang ${ }^{1 *}$, Yi Du ${ }^{1,2^{*}}$, Lei Jiang ${ }^{2,3,4}$ and Shi-Xue Dou ${ }^{1,2}$
}

Noble metals have been widely applied as catalysts in chemical production, energy conversion, and emission control [1-3], but their high cost and scarcity are major obstacles for any large-scale practical applications. It is therefore of great interest to explore new active material systems that require less mass loading of noble metal catalysts but with even better performance. Recently, intense research has been devoted towards downsizing the noble metals into single-atom catalysts (SACs) [4,5]. SACs, with single-atom active centers, were first reported by Qiao et al. [4]. They synthesized a single Pt atom catalyst supported on $\mathrm{FeO}_{x}\left(\mathrm{Pt}_{1} / \mathrm{FeO}_{x}\right)$, which offered extremely high efficiency on an atomic percent basis and showed excellent performance towards CO oxidation.

There is no doubt that SACs offer superior performance towards some catalytic reactions, because of the unsaturated coordination environment of their metal species and highly active valence electrons. In addition, the surface free energy of metal species reaches a maximum in SACs [6-10]. Nevertheless, it is worth noting that the catalytic selectivity plays an important part in catalytic reactions, and the SACs may limit application in some multi-electron catalytic processes [5]. In this case, we propose that if single atoms could assemble to monatomic layer clusters (mALCs), they would exhibit higher catalytic activity and selectivity than SACs. Unlike SACs [11-13], the mALCs materials are expected to be stable and to keep its structure under realistic catalytic conditions, which outperformes well-defined monatomic layer.

The coordination environment of mALCs will be more saturated than that of single atoms, which indicates $m$ ALCs materials could be more stable during catalytic reaction. In addition, under the realistic reaction condition, the atoms of $m$ ALCs could work in synergy to catalyze the reaction. For example, neighboring $\mathrm{Pt}$ monomers showed better catalytic performance and lower activation energy than single $\mathrm{Pt}$ atoms towards $\mathrm{CO}_{2}$ reduction; meanwhile, these neighboring $\mathrm{Pt}$ monomers did not aggregate during the catalytic reaction [8]. It is generally believed that the support materials could affect electronic configuration of noble metal atoms by rearranging the molecular orbitals. This phenomenon results in modification of local charge density in catalyst surface. Based on the combination ways between noble metal atoms and matrix atoms, there are two models to describe the interaction between noble metal atoms and matrix atoms corresponding to the simulating charge density maps, as shown in Fig. 1. For SACs, if the single noble atom is anchored by three or four matrix atoms, its charge density will be affected only by matrix atoms. Its low-coordination environment results in a high activity. In contrast, if the single atoms assemble to monatomic layer clusters (for example, two atoms or four atoms), their energy states and charge density will be determined by both matrix atoms and neighboring noble atoms through the hybridization. Moreover, the energy of the highest occupied molecular orbital (HOMO) could be close to that of adsorbed species (such as $\mathrm{O}_{2}$ and $\mathrm{CO}_{2}$ ), which will facilitate charge transfer and decrease the reaction energy. Compared with nanoparticles (shown at the right end of Fig. 1), the noble atoms in the mALCs

\footnotetext{
${ }^{1}$ Institute for Superconducting and Electronic Materials, Australian Institute of Innovative Materials, University of Wollongong, Innovation Campus, Squires Way, North Wollongong, New South Wales 2500, Australia

${ }^{2}$ BUAA-UOW Joint Research Centre, Beihang University, Beijing 100191, China

${ }^{3}$ Key Laboratory of Bio-Inspired Smart Interfacial Science and Technology of the Ministry of Education, School of Chemistry and Environment, Beihang University, Beijing 100191, China

${ }^{4}$ Laboratory of Bio-inspired Smart Interface Science, Technical Institute of Physics and Chemistry, Chinese Academy of Sciences, Beijing 100191, China

*Corresponding authors (emails: yunxiao@uow.edu.au (Wang YX); ydu@uow.edu.au (Du Y))
} 


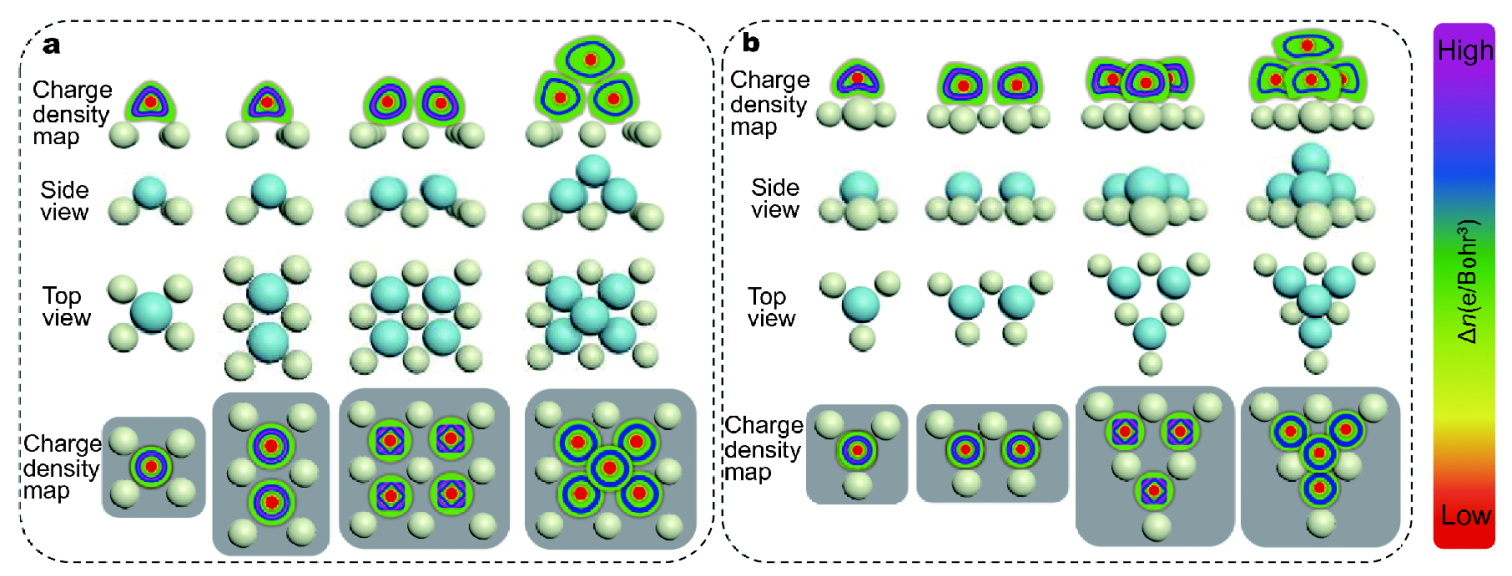

Figure 1 Two different types of noble metal atoms (blue) anchored on matrix atoms (white), and the corresponding simulated charge density map.

also have a lower coordination number and higher surface free energy. These two features of mALCs lead to their higher activity and selectivity than those of SACs and conventional nanomaterials.

It is well-known that catalytic reactions take place on the surface of catalysts $[14,15]$. Even with the same element, different crystal planes could possess a very different work function and catalytic activity. For example, the Pt (111) surface shows higher activity than the Pt (100) and Pt (110) towards the oxygen reduction reaction (ORR) in $\mathrm{HClO}_{4}$ solution [16]. This indicates that the multi-electron transfer process involved in the ORR on different Pt crystal planes will result in different reaction barriers. This is not only the case for monometallic catalysts, but also for bimetallic catalysts. For instance, Stamenkovic et al. [17] demonstrated that the $\mathrm{Pt}_{3} \mathrm{Ni}$ (111) surface is more active than the $\mathrm{Pt}_{3} \mathrm{Ni}$ (100) surface or the $\mathrm{Pt}_{3} \mathrm{Ni}$ (110) surface towards the ORR, as shown in Fig. 2.

It is suggested that the surface structure of nanomaterials directly determines their performance in catalytic reactions. The high-index facet nanomaterials, with a large density of atomic steps and dangling bonds have demonstrated much enhanced specific activity compared to low-index Pt surfaces [18]. Although the high-index facet nanomaterials have high specific activity, it remains a challenge to decrease their size (usually $\sim 100 \mathrm{~nm}$ ) to meet commerical demands (commercial $\mathrm{Pt} / \mathrm{C}$ catalyst particles are normally $\sim 3 \mathrm{~nm}$ in size). Significant efforts have been devoted to the synthesis of small nanomaterials with controllable high-index facets. For example, Liu et al. [19] developed a novel electrochemically seed-mediated method to synthesize sub $-10 \mathrm{~nm}$ tetrahexahedral $\mathrm{Pt}$ nanomaterials with high-index facets, possessing higher mass activity than commercial $\mathrm{Pt} / \mathrm{C}$ towards ethanol



Figure 2 Influence of the surface morphology and electronic surface properties on the kinetics of the ORR. Rotating ring-disk electrode measurements of the ORR in $\mathrm{HClO}_{4}\left(0.1 \mathrm{~mol} \mathrm{~L}^{-1}\right)$ at $333 \mathrm{~K}$ and 1,600 revolutions per minute on $\mathrm{Pt}_{3} \mathrm{Ni}(h k l)$ surfaces are shown as compared to the corresponding $\mathrm{Pt}(h k l)$ surfaces. Reprinted with permission from Ref. [17], Copyright 2007, the American Association for the Advancement of Science (AAAS).

electrooxidation to $\mathrm{CO}_{2}$. From the dependence of the structure-catalytic performances, we believe that synthesis of abundant active step sites is an effective approach to enhancing catalytic performance and avoiding the occurrence of side effects. Based on this consideration, SACs may not meet this requirement.

Clusters containing only a few atoms exhibit unique and unexpected properties, which could serve as individual active sites in the catalytic process. On the basis of this behavior, clusters have attracted much attention because every atom could have a substantial effect on the catalytic selectivity and activity. Even a subtle change in cluster size may lead to a significant change in catalytic performances [20]. For example, Kaden et al. [21] studied $\mathrm{Pd}_{n}$ clusters $(n \leq 25)$ on rutile $\mathrm{TiO}_{2}(110)$ for their perfor- 
mance towards $\mathrm{CO}$ oxidation. They found that $\mathrm{Pd}_{2}$ and $\mathrm{Pd}_{20}$ clusters exhibited higher relative activity because of their unstable valence shell, which strongly depends on the size and electron number of the cluster, as shown in Fig. 3. The results also demonstrated that single Pd atoms are not active towards $\mathrm{CO}$ oxidation, and their production of $\mathrm{CO}_{2}$ is equal to that of rutile $\mathrm{TiO}_{2}(110)$. A study on the oxidation of $\mathrm{CO}$ using $\mathrm{Au}_{n}$ cluster catalysts demonstrates that $\mathrm{Au}_{8}$ on $\mathrm{MgO}(001)$ is the smallest $\mathrm{Au}$ cluster active towards $\mathrm{CO}$ oxidation at $140 \mathrm{~K}$ [22]. This study also reveals that the support will influence the electronic properties of $\mathrm{Au}_{8}$, which can enhance the catalytic performance towards $\mathrm{CO}$ oxidation. In order to reach a better understanding of the fundamental properties of catalytic active sites on the atomic scale, combined experimental and computational investigations have been undertaken $[23,24]$. Clusters, which are relatively small in size, are suitable for computational modelling because of such systems being quite unambiguous.

Nevertheless, there are some limitations on the preparation of well-defined clusters in industrial-scale quantities. In 2017, atomic Au layered clusters $(\sim 2 \mathrm{~nm})$

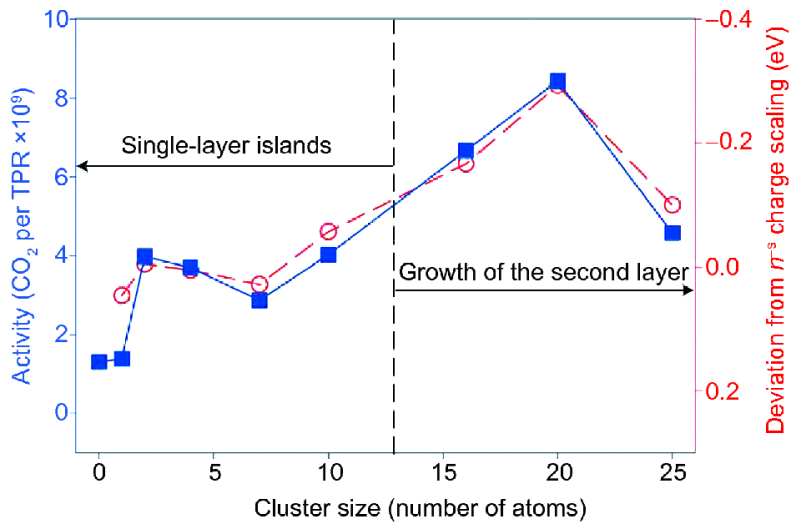

Figure $3 \mathrm{CO}$ oxidation activity observed during temperatureprogrammed reduction (TPR) (left axis, solid squares) compared with shifts in the Pd $3 \mathrm{~d}$ binding energy, relative to expectations from smooth bulk scaling (right axis, open circles), as a function of cluster size. Reprinted with permission from Ref. [21], Copyright 2009, AAAS.

supported on a-MoC exhibited higher water-gas shift reaction activity than single atom $\mathrm{Au}$ [25], as shown in Fig. 4. Meanwhile, the atomic-layered Au clusters keep
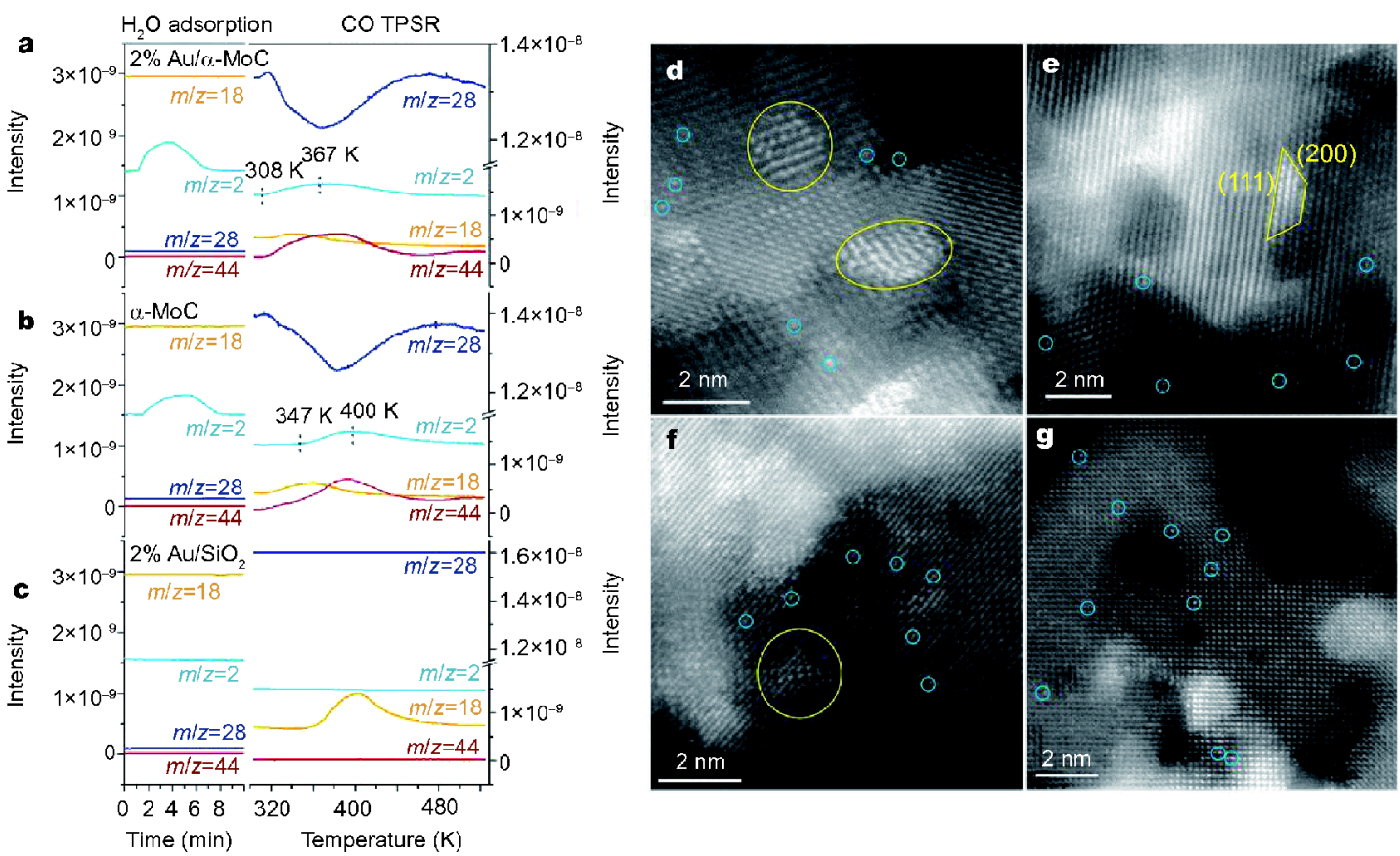

Figure 4 Mechanism study and electron microscopy characterization. Water adsorption (at $303 \mathrm{~K}$ ) followed by CO-temperature programmed surface reaction (TPSR) on $2 \% \mathrm{Au} / \mathrm{\alpha}-\mathrm{MoC}(\mathrm{a}), \alpha-\mathrm{MoC}(\mathrm{b})$, and $2 \% \mathrm{Au} / \mathrm{SiO}_{2}$ (c). Signals of $\mathrm{H}_{2}$ (mass to charge ratio, $\left.m / z=2\right), \mathrm{H}_{2} \mathrm{O}(\mathrm{m} / z=18), \mathrm{CO}(\mathrm{m} / z=28)$, and $\mathrm{CO}_{2}(m / z=44)$ were detected. ( $\mathrm{d}$ and e) High-resolution high angle annular dark field-scanning transmission electron microscopy (HAADF-STEM) images of $2 \% \mathrm{Au} / \mathrm{a}-\mathrm{MoC}$ fresh catalyst, with single atoms of Au marked in blue circles and Au layered-structures highlighted in yellow. (f) HAADFSTEM image of $2 \% \mathrm{Au} / \mathrm{\alpha}-\mathrm{MoC}$ catalyst after reaction, in which the sample still contains both single-atom Au and Au layered-clusters. (g) HAADFSTEM image of the $\mathrm{NaCN}$ leached $2 \% \mathrm{Au} / \mathrm{a}-\mathrm{MoC}$ catalyst, showing predominantly single atom $\mathrm{Au}$, most of which overlaps with Mo sites in the support lattice. Reprinted with permission from Ref. [25], Copyright 2017, AAAS. 
their morphology up to $473 \mathrm{~K}$ during water-gas shift reaction. On the basis of the above knowledge, we believe that mALCs would exhibit higher activity and selectivity than other nanomaterials, even SACs. To the best of our knowledge, currently, mALCs are still in their research infancy but are likely to lead to great opportunities and challenges in the fields of chemistry, materials science, and nanotechnology.

\section{Received 24 April 2018; accepted 26 June 2018;} published online 10 July 2018

1 Grirrane A, Corma A, García H. Gold-catalyzed synthesis of aromatic azo compounds from anilines and nitroaromatics. Science, 2008, 322: 1661-1664

2 Rodriguez JA, Ma S, Liu P, et al. Activity of $\mathrm{CeO}_{x}$ and $\mathrm{TiO}_{x}$ nanoparticles grown on $\mathrm{Au}(111)$ in the water-gas shift reaction. Science, 2007, 318: 1757-1760

3 Gilroy KD, Ruditskiy A, Peng HC, et al. Bimetallic nanocrystals: syntheses, properties, and applications. Chem Rev, 2016, 116: 10414-10472

4 Qiao B, Wang A, Yang X, et al. Single-atom catalysis of CO oxidation using $\mathrm{Pt}_{1} / \mathrm{FeO}_{x}$. Nat Chem, 2011, 3: 634-641

5 Yang XF, Wang A, Qiao B, et al. Single-atom catalysts: a new frontier in heterogeneous catalysis. Acc Chem Res, 2013, 46: 17401748

6 Bayatsarmadi B, Zheng Y, Vasileff A, et al. Recent advances in atomic metal doping of carbon-based nanomaterials for energy conversion. Small, 2017, 13: 1700191

7 Tsuji Y, Yamamoto K, Yamauchi K, et al. Near-infrared lightdriven hydrogen evolution from water using a polypyridyl triruthenium photosensitizer. Angew Chem Int Ed, 2018, 57: 208-212

$8 \mathrm{Li} \mathrm{H}$, Wang L, Dai Y, et al. Synergetic interaction between neighbouring platinum monomers in $\mathrm{CO}_{2}$ hydrogenation. Nat Nanotechnol, 2018, 13: 411-417

9 Guo X, Fang G, Li G, et al. Direct, nonoxidative conversion of methane to ethylene, aromatics, and hydrogen. Science, 2014, 344: 616-619

10 Jones J, Xiong H, DeLaRiva AT, et al. Thermally stable single-atom platinum-on-ceria catalysts via atom trapping. Science, 2016, 353: 150-154

11 Deng D, Chen X, Yu L, et al. A single iron site confined in a graphene matrix for the catalytic oxidation of benzene at room temperature. Sci Adv, 2015, 1: e1500462

12 Liu P, Zhao Y, Qin R, et al. Photochemical route for synthesizing atomically dispersed palladium catalysts. Science, 2016, 352: 797800

13 Wang H, Wang Q, Cheng Y, et al. Doping monolayer graphene with single atom substitutions. Nano Lett, 2012, 12: 141-144

14 Zhang BW, Sheng T, Wang YX, et al. Platinum-cobalt bimetallic nanoparticles with Pt skin for electro-oxidation of ethanol. ACS Catal, 2017, 7: 892-895

15 Liu L, Corma A. Metal catalysts for heterogeneous catalysis: from single atoms to nanoclusters and nanoparticles. Chem Rev, 2018, 118: 4981-5079

16 Guo S, Zhang S, Sun S. Tuning nanoparticle catalysis for the oxygen reduction reaction. Angew Chem Int Ed, 2013, 52: 85268544

17 Stamenkovic VR, Fowler B, Mun BS, et al. Improved oxygen reduction activity on $\mathrm{Pt}_{3} \mathrm{Ni}(111)$ via increased surface site availability. Science, 2007, 315: 493-497

18 Tian N, Zhou ZY, Sun SG, et al. Synthesis of tetrahexahedral platinum nanocrystals with high-index facets and high electrooxidation activity. Science, 2007, 316: 732-735

19 Liu S, Tian N, Xie AY, et al. Electrochemically seed-mediated synthesis of sub-10 nm tetrahexahedral Pt nanocrystals supported on graphene with improved catalytic performance. J Am Chem Soc, 2016, 138: 5753-5756

20 Tyo EC, Vajda S. Catalysis by clusters with precise numbers of atoms. Nat Nanotechnol, 2015, 10: 577-588

21 Kaden WE, Wu T, Kunkel WA, et al. Electronic structure controls reactivity of size-selected $\mathrm{Pd}$ clusters adsorbed on $\mathrm{TiO}_{2}$ surfaces. Science, 2009, 326: 826-829

22 Sanchez A, Abbet S, Heiz U, et al. When gold is not noble: nanoscale gold catalysts. J Phys Chem A, 1999, 103: 9573-9578

23 Jin $\mathrm{H}$, Guo C, Liu X, et al. Emerging two-dimensional nanomaterials for electrocatalysis. Chem Rev, 2018, doi:10.1021/acs. chemrev.7b00689

24 Huber B, Koskinen P, Häkkinen $\mathrm{H}$, et al. Oxidation of magnesiasupported Pd-clusters leads to the ultimate limit of epitaxy with a catalytic function. Nat Mater, 2006, 5: 44-47

25 Yao S, Zhang X, Zhou W, et al. Atomic-layered Au clusters on a$\mathrm{MoC}$ as catalysts for the low-temperature water-gas shift reaction. Science, 2017, 357: 389-393

Acknowledgements This research was supported by the Australian Research Council (ARC) (DE170100928 and DP170101467), the Commonwealth of Australia through the Automotive Australia $2020 \mathrm{Co}$ operative Research Centre (AutoCRC), and Baosteel-Australia Joint Research and Development Center (BA14006). The authors acknowledge the use of the facilities at the UOW Electron Microscopy Centre funded by ARC Grants (LE0882813 and LE0237478) and Dr. Tania Silver for her critical reading.

Author contributions Jiang L proposed the concept of monatomic layer clusters. All authors contributed to the general discussion.

Conflict of interest The authors declare no conflict of interest. 

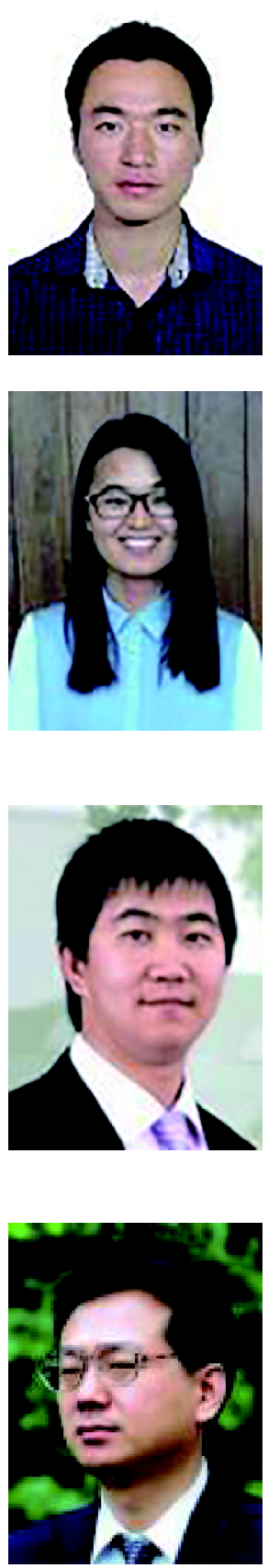

Bin-Wei Zhang received his bachelor degree and master degree in the College of Chemistry and Chemical Engineering, Xiamen University in 2012 and 2015, respectively. He is a PhD candidate at the University of Wollongong. His current research interest is renewable energy storage and conversion, including electrocatalysis and lithium/sodium sulfur batteries.

Yun-Xiao Wang is a Research Fellow at the Institute for Superconducting and Electronic Materials (ISEM), University of Wollongong (UOW). She received her bachelor degree (2008) from Hebei Normal University and her master degree (2011) from Xiamen University. She obtained her PhD degree from the University of Wollongong in 2015. Her current research interest is renewable energy storage and conversion, including electrocatalysis, lithium/sodium-ion batteries, and lithium/sodium sulfur batteries.

Yi Du is a Senior Research Fellow at the Institute for Superconducting and Electronic Materials (ISEM), UOW. He received his bachelor degree (2004) and master degree (2007) from Beihang University, China. He obtained his PhD degree from the University of Wollongong in 2011. His current research interest is exploration and development of novel two-dimensional materials and their surface physics and chemistry with scanning probe microscopies (STM, AFM and SNOM).

Lei Jiang is currently a Professor at the Institute of Chemistry, Chinese Academy of Sciences (ICCAS), and Dean of the School of Chemistry and Environment, Beihang University. He received his BSc degree (1987), MSc degree (1990), and $\mathrm{PhD}$ degree (1994) from Jilin University, China (Jintie Li's group). He then worked as a postdoctoral fellow in Professor Akira Fujishima's group in Tokyo University. In 1996, he worked as a senior researcher in Kanagawa Academy of Sciences and Technology under Professor Kazuhito Hashimoto's group. He joined ICCAS as part of the Hundred Talents Program in 1999. He was elected the academician of the Chinese Academy of Sciences in 2009 and the Academy of Sciences for the Developing World in 2012. His scientific interests are focused on bioinspired, smart, multiscale interfacial (BSMI) materials.

\section{新型的单原子层团簇催化剂}

张斌伟 ${ }^{1}$, 任龙 ${ }^{1}$ 王云晓 ${ }^{*}$, 杜轶 ${ }^{1,2^{*}}$, 江雷 ${ }^{2,3,4}$, 窦士学 ${ }^{1,2}$

摘要 “单原子层团簇”催化剂这一新概念, 不同于单原子催化剂和传统的纳米颗粒催化, 是由单原子建造新型的二维单原子层催化剂. 单 原子层团簇催化剂的活性中心明确, 且原子间的相互作用会极大提高催化反应的选择性. 因此该催化剂材料不仅具有优异的催化性能, 还 具有良好的选择性. 基于此, 作者同时分析和指出了未来的单原子层团簇催化剂的可能重点研究方向以及挑战. 